\title{
La tecnología digital como \\ herramienta para la democratización de ideas matemáticas poderosas*
}

Digital Technology as a Tool for the Democratization of Powerful Mathematical Ideas

A tecnologia digital como ferramenta para a democratização de ideias matemática poderosas

\section{Santiago Palmas-Pérez ** (iD) orcid.org/0000-0003-1175-5938}

Artículo de reflexión derivado de investigación

Revista Colombiana de Educación, N.7 74. Primer semestre de 2018, Bogotá, Colombia.

Para citar este artículo: Palmas, S. (2018). La tecnología digital como herramienta para la democratización de ideas matemáticas poderosas. Revista Colombiana de Educación, (74), 109-132.

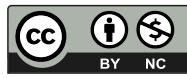

Recibido: 09/12/2016

Evaluado: 08/02/2017

El presente trabajo deriva parcialmente de mi investigación doctoral. Agradezco a la doctora Teresa Rojano Ceballos su dirección y apoyo.

** Doctor en Ciencias. Profesor visitante asociado al Departamento de Estudios Culturales. Lerma, Estado de México, México. Correo electrónico: s.palmas@correo.ler.uam.mx 


\title{
Resumen
}

En este artículo se analiza el papel de la tecnología como medio de acceso a ideas matemáticas poderosas (Skovsmose y Valero, 2009) en la educación matemática para jóvenes y adultos. En particular, se busca responder a la pregunta: ¿puede la tecnología fungir como puente entre las prácticas matemáticas culturales y su modelización en favor de un acceso democrático a ideas matemáticas poderosas? Se plantea la posibilidad de que la tecnología se utilice como herramienta democratizadora de dichas ideas, siempre y cuando se aborden necesidades educativas con base en concepciones matemáticas previas establecidas por los propios educandos. En el escrito se presenta un desarrollo didáctico, basado en la teoría de situaciones didácticas (Brousseau, 1997), así como las decisiones que condujeron al desarrollo de una herramienta tecnológica ad hoc, que sirviera como puente hacia ideas matemáticas poderosas. Se muestra cómo ambos diseños promovieron la construcción de conceptos e ideas matemáticas poderosas al tiempo que se discute sobre la posibilidad de usar la tecnología como herramienta democratizadora del conocimiento.

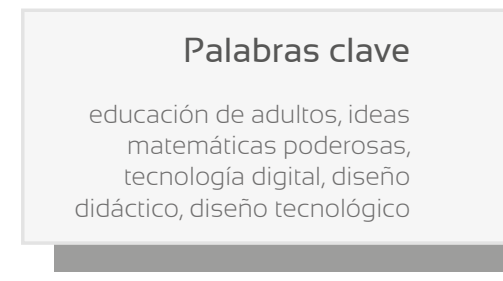

\section{Keywords}

adult education; powerful mathematical ideas; digital technology; didactic design; technological design

\begin{abstract}
In this article we analyze the role of technology as a means of accessing powerful mathematical ideas (Skovsmose \& Valero, 2009) in mathematics education for young people and adults. More particularly, we seek to answer: Can technology act as a bridge between cultural mathematical practices and modeling in favor of a democratic access to powerful mathematical ideas? The possibility of technology being used as a democratizing too for these ideas is proposed, provided that educational needs are addressed according to previous mathematical conceptions established by the students themselves. In this paper, we present a didactic development, based on the theory of didactictal situations (Brousseau, 1997), as well as the decisions that led to the development of an ad hoc technological tool, serving as a bridge to powerful mathematical ideas. We show how both designs promoted the construction of powerful mathematical ideas and concepts while discussing the possibility of using technology as a democratizing tool of knowledge.
\end{abstract}

\begin{abstract}
Resumo
Neste artigo é analisado o papel da tecnologia como meio de acesso a ideias matemáticas poderosas (Skovsmose \& Valero, 2009) na educação matemática para jovens e adultos. O trabalho tenta responder à pergunta: pode a tecnologia funcionar como ponte entre as práticas matemáticas culturais e a sua modelização para um acesso democrático às ideias matemáticas poderosas? Pondera-se a possibilidade que a tecnologia seja utilizada como ferramenta democratizadora dessas ideias, sempre que sejam abordadas as necessidades educativas com base nas concepções matemáticas prévias estabelecidas pelos educandos. No escrito, apresenta-se um desenvolvimento didático, baseado na teoria de situações didáticas (Brousseau, 1997), assim como as decisões que encorajaram o desenvolvimento de uma ferramenta tecnológica ad hoc, que funciona como ponte para as ideias matemáticas poderosas. Apresenta-se como os dois projetos promoveram a construção de concepções e ideias matemáticas poderosas. Ao mesmo tempo, se debate sobre a possibilidade de utilizar a tecnologia como ferramenta democratizadora do conhecimento.
\end{abstract}

\section{Palavras-chave}

educação de adultos: ideias matemáticas

poderosas; tecnologia

digital; desenho didático; desenho tecnológico 


\section{Introducción}

México es uno de los países con mayores contrastes sociales y económicos del mundo. Basta con caminar atentamente por la calle y reconocer en sus recovecos la coexistencia de una minoría que goza de viviendas dignas, servicios de salud de primer mundo, y amplias oportunidades educativas, con una gran mayoría que vive en condiciones de pobreza y escasez. Los contextos de vida de una minoría contrastan con un sistema de salud saturado, transporte inseguro, escuelas sobrepobladas y una temprana incorporación al trabajo. Este panorama prevalece en Latinoamérica.

La desigualdad se traduce en grandes diferencias en las oportunidades de vida y bienestar general que no solo provienen de la distribución disímil de los recursos económicos, sino también del conocimiento. Existe una direccionalidad, un flujo de conocimiento e información que está disponible únicamente para aquéllos que tienen determinado capital social, cultural y económico. En general, las estructuras sociales actuales hacen que este flujo no sea accesible para estratos económicamente bajos de nuestra sociedad porque, como señalan Skovsmose y Valero, "todos los excluidos pertenecen a áreas estructuralmente irrelevantes en la sociedad informacional" (2009, p. 28). En palabras de Flecha:

... el conocimiento priorizado por las nuevas formas de vida se distribuye de manera desigual entre los individuos, de acuerdo con el grupo social, el género, el grupo étnico y la edad. Al mismo tiempo, el conocimiento que poseen los grupos marginados no se tiene en cuenta, incluso si es más rico y más complejo que el conocimiento priorizado. Se da más a aquellos que tienen más y menos a aquellos que tienen menos, cerrando un círculo de desigualdad cultural. (1999, p. 67).

Existen muchos jóvenes y adultos que no acceden a estos flujos de conocimiento por el hecho de no encontrar cabida dentro de una cultura escolar. Las desigualdades en el acceso a conocimientos e información representan oportunidades para repensar la vida diaria y la posibilidad de transformar nuestra realidad. La educación con jóvenes y adultos debería formar parte de un esfuerzo por paliar las desigualdades en el acceso a oportunidades educativas en pro de una sociedad que favorezca la democratización del conocimiento; cualquiera y en cualquier sentido, teniendo en cuenta lo que las personas saben y quieren saber.

¿Cómo podemos afrontar las desigualdades de acceso al conocimiento? Un camino posible, es ampliando la mirada hacia diferentes formas de conocimiento, por ejemplo, lo matemático, reconociendo que de las prácticas sociales matemáticas surgen métodos que se pueden retomar para construir nuevos procesos matemáticos. Además, es necesario encontrar medios que den preponderancia a la acción sobre el mero 
intercambio de información. De lo anterior, surge la propuesta de indagar el papel de la tecnología como medio de acceso a ideas matemáticas que dialogue entre lo que los adultos saben y lo que quieren conocer.

Inicialmente se plantea la importancia de revelar el aspecto matemático en la educación de jóvenes y adultos, y discutiendo el concepto de numeralismo. Posteriormente, se presentan las ideas matemáticas poderosas como concepto que visibiliza las relaciones democráticas en la educación matemática. Planteando así el panorama, se analiza el papel de la tecnología como medio de acceso a dichas ideas matemáticas poderosas, sus beneficios y desafíos. Por último, se muestra un diseño didáctico y tecnológico que aborda necesidades educativas geométricas de adultos, como experiencia práctica de democratización del conocimiento matemático.

\section{Sobre la importancia del numeralismo en la educación matemática de jóvenes y adultos}

La educación de jóvenes y adultos (EDJA) con baja escolaridad es una línea de investigación educativa poco estudiada. Desde hace casi dos décadas, en el estado de la cuestión de la educación de adultos Schmelkes y Kalman (1996) plantean que:

\footnotetext{
Aunque siempre se dice que la alfabetización atiende la lectoescritura y el cálculo básico, a éste se le da mucha menor importancia, a pesar de que entre los adultos es sentido quizá como una mayor necesidad que la de aprender a leer y a escribir. La impotencia ante el engaño en las transacciones comerciales o en los acuerdos laborales es una razón frecuentemente planteada por los adultos analfabetas al referirse a su necesidad de aprender cálculo básico. (p. 22).
}

Desde la época del estudio citado se han hecho algunos avances en la educación matemática con adultos de nivel educativo medio y medio superior, pero aún no se ha consolidado el trabajo de investigación en torno a la educación matemática de jóvenes y adultos (EMDJA) de baja o nula escolaridad. Más bien, lo que se observa es una tendencia a investigar procesos de enseñanza y aprendizaje en el campo de la educación matemática para niños, reduciendo sustancialmente el número de estudios que tratan procesos educativos fuera de la escuela o con poblaciones que han tenido poco acceso a ella. Este trabajo busca contribuir a la discusión sobre la EMDJA a partir de un trabajo cualitativo cuya mirada se centra en los adultos de baja escolaridad y las posibilidades que la tecnología digital ofrece en este contexto educativo. 


\section{Sobre la EMDJA}

Las matemáticas, como parte de la ciencia y del conocimiento, son un elemento cultural que permite participar en contextos sociales y políticos, por ejemplo, en actividades laborales y escolares. En una "sociedad informacional" (Castells, 1996) tanto la ciencia como la tecnología desempeñan un rol activo, considerando que "el impacto de la tecnología va más allá de la producción industrial, y, de hecho, afecta estructuras políticas, económicas, sociales y culturales" (Skovsmose y Valero, 2009, p. 26); así vemos que las matemáticas no son elementos neutrales en dichas estructuras.

Para entender estos fenómenos sociales en la educación matemática, es necesario ampliar la mirada de lo que significa investigar la educación matemática, e incluir la relación en un espectro amplio que abarque lo extraescolar. Por ello, con la preocupación de abordar la EMDJA de manera que incluya aspectos sociales, culturales y políticos, aparece el término numeralismo o alfabetización matemática ${ }^{1}$.

En el presente estudio, reconociendo los ejercicios del poder en las actividades matemáticas, el numeralismo se considera como un proceso que incluye acciones visibles e invisibles, dentro de una actividad cultural que permite posicionarse de diferente manera en el mundo. Estos procesos de construcción de una cultura matemática implican apropiarse no solo de un repertorio de conceptos, sino también de ideas matemáticas poderosas (Skovsmose y Valero, 2009) que permitan a la gente visualizarse y participar de diferente manera ante situaciones cotidianas: el "ser más" del que habla Freire. Este término se puede ampliar con la definición de alfabetización matemática de Skovsmose (1999, p. 26):

... el papel crítico de las matemáticas en la formatación del mundo social, la competencia crítica del ciudadano requiere de un cierto grado de competencia matemática para poder tomar una distancia y juzgar las decisiones de los gobernantes, cuando esas decisiones se basan en sistemas y modelos matemáticos expertos. La competencia matemática democrática no es sólo saber matemáticas para poseer una serie básica de conocimientos requeridos hoy en día en el mercado laboral, sino que es poner ese conocimiento matemático en juego para cuestionar a las autoridades y, por lo tanto, poder hacer frente a la injusticia. (Valero, Andrade-Molina y Montecino, 2015, p. 291).

Reflexionar en torno a estos procesos de poder, junto con el diseño propio de actividades y el uso de conceptos matemáticos, favorecerá el acceso a ideas matemáticas poderosas, siempre y cuando haya un

1 Algunos autores lo han descrito como numeracy (por ejemplo, Street, Baker y Tomlin, 2008), matheracy (por D’Ambrossio, 1996) o mathemacy (por Skovsmose, 1999). 
momento educativo intencionado que incluya las prácticas sociales de los educandos como punto de partida y un medio (didáctico y tecnológico, por ejemplo) que organice el tránsito hacia dichas ideas.

Así, el estudio que se resume en el presente artículo comenzó por una indagación de lo que los adultos quieren conocer, lo que saben, y no desde lo que se supone que ignoran. Este trabajo pretende abarcar una parte importante de la EDJA: la EMDJA, no calculista ni utilitarista, sino constructora de puente entre las prácticas culturales matemáticas e ideas matemáticas poderosas, teniendo como medio/mediador la tecnología digital y una situación didáctica.

Desde la definición de numeralismo presentada y la comprensión del fenómeno educativo relativo a la EMDJA, discuto a continuación la importancia de considerar a las ideas matemáticas poderosas como acciones no observables a las que es posible acceder.

\section{Sobre las ideas matemáticas poderosas}

Skovsmose y Valero (2009) señalan la existencia de ideas matemáticas poderosas detrás de las acciones observables. Esta noción surge de la necesidad de hacer visibles los ejercicios de poder que permiten participar en ciertas actividades socioculturales al usar las matemáticas. Para reconocer el carácter de poder en estas ideas, con relación a la opresión social, cultural y económica, es útil recordar que las matemáticas se usan constantemente para excluir de trabajos a personas que lo necesitan; es aquí donde "lo poderoso significa tanto como afirmar que puede ejercer poder" (Skovsmose y Valero, 2009, p. 36). Es decir, quien posee dichas ideas matemáticas puede ejercer poder ante otras personas. Por lo anterior surge la idea de democratizar el acceso a ideas matemáticas poderosas, entendido como "la posibilidad de ingresar a un tipo de educación matemática que favorezca la consolidación de las relaciones sociales democráticas" (Skovsmose y Valero, 2012, p. 48) con la finalidad de construir junto con el educando oportunidades de visualizar oportunidades de acción ante fenómenos de desigualdad. Skovsmose y Valero, así como Rojano, exponen cuatro perspectivas para entender las ideas matemáticas poderosas:

» Desde un punto de vista lógico, las ideas matemáticas ligadas a la abstracción proporcionan nuevas maneras de comprender el mundo dentro de un conjunto existente de diferentes conceptos, "un poder intrínseco dentro de las matemáticas" (Skovsmose y Valero, 2009, p. 37).

» Desde un punto de vista psicológico el poder se determina en relación con las potencialidades de aprendizaje. En este sentido, las definiciones de ideas matemáticas poderosas de Rojano (2002) son las que favorecen el acceso a un pensamiento más abstracto, 
formal y complejo. Estas ideas se fundamentan en una noción de tránsito entre niveles de cognición de menor a mayor nivel de abstracción. Algunas de estas nociones de tránsito son²:

- De la aritmética al álgebra. Por ejemplo, el signo [=] en la aritmética se refiere a una acción que transforma el lado izquierdo en lo escrito en el derecho; sin embargo, en el álgebra el mismo símbolo puede representar equivalencia, igualdad o una relación o función,

- De lo específico a lo general. Por ejemplo, cuando se hacen predicciones del número de puntos en una secuencia de ellos (por ejemplo, trabajando en hojas de cálculo).

- De lo informal a lo formal. Por ejemplo, la idea de ir refinando y sistematizando la resolución de problemas por medio del ensayo y error.

- Del dibujo a la figura geométrica. Se asemeja a la idea de pasar de lo perceptual a lo conceptual.

- En general, hacia un pensamiento más abstracto (Rojano, 2002).

La autora elabora un extenso análisis de estudios con adolescentes, en los cuales se estudian los procesos cognitivos para precisar las ideas matemáticas poderosas como aquellas que permiten procesos de transición entre lo concreto y lo abstracto.

» Punto de vista cultural: "Relacionado con las oportunidades que tienen los estudiantes de participar en las prácticas de una comunidad más pequeña o de la sociedad más amplia" (Skovsmose y Valero, 2009, p. 43). Son las redes de relaciones y significados construidos socialmente que permiten involucrarse en sociedad en ciertas acciones culturales.

» Punto de vista sociológico. Se relaciona con el alcance de ciertos recursos matemáticos para poder actuar en sociedad. Por ejemplo, el uso de las matemáticas como herramientas descriptivas o de creación de hipótesis. Esta idea matemática poderosa incluye una visión del mundo en donde se sitúa el uso de la herramienta. (Skovsmose y Valero, 2009, pp. 36-48)

Kaput (1994) consideraba que la democratización del conocimiento matemático podría venir desde edades tempranas, por ejemplo, acercando el cálculo a niños. Esto cuestionaba la idea de que la matemática avanzada está reservada para una población que llega a la universidad y a ciertas carreras. Para Kaput, reducir la edad en la que se tiene acceso a ideas matemáticas poderosas era una forma de democratizar el conocimiento. En el presente estudio, la democratización del conocimiento no está pensada en el acceso 
temprano a ideas matemáticas, sino en el fomento del acceso a ideas matemáticas poderosas para sectores amplios de la sociedad, como las personas con baja escolaridad, sector que tradicionalmente ha sido excluido de ellas.

\section{Sobre el papel de la tecnología}

Teniendo en cuenta el panorama anterior, ¿qué papel puede tener la tecnología como herramienta de acceso a ideas matemáticas poderosas?

\section{Acceso}

En primer lugar, para poder responder a esta pregunta es necesario reconocer que el papel de la tecnología no puede estar supeditado a la disponibilidad de materiales, sino que involucra analizar los procesos de uso que dan sentido a las actividades de los adultos en procesos educativos y sus posibilidades de participar en el mundo. Es común usar el término tecnologías digitales (O TD) para diferenciar esta posición de la que subyace al término tecnologías de la información y la comunicación (TIC). Este término, TIC, proviene de modelos económicos posindustriales; Warschauer (2003) denomina a esta etapa la "Tercer Revolución Industrial", mientras que Castells (2000) la llama "informacionalismo". Sobre esta "revolución" habló Herbert Simon:

Estamos en los primeros años de una revolución en el procesamiento de la información que muestra signos de ser tan fundamental como la anterior revolución energética. Posiblemente, le podríamos denominar "Tercera Revolución de la Información" (La primera producida por el lenguaje escrito y la segunda por el libro impreso). Esta tercera revolución, que empezó más de un siglo atrás, incluye la computadora entre otras cosas. La tecnología de la información abarca un amplio rango de procesos de almacenamiento de información, para copiarlo o transmitirlo. (1977, p. 1186).

Tanto Castells como Warschauer y Simon notan en esta aparición de nuevas herramientas una cargada tendencia hacia la información como culto. Este foco "informacionalista" se mantuvo hasta 1994, cuando se acuñó el término de la "nueva economía" (Laursen, 2004) posteriormente formalizado e institucionalizado por la Organización para la Cooperación y el Desarrollo Económicos (OCDE) en 1998³. Así, el término tıc refleja una

3 https://stats.oecd.org/glossary/detail.asp?ID=3038. Esta página es un glosario de términos de la ocde. En su definición de "The Information, Communication Technology sector (ICT)", en la sección de contexto se lee: "The current oecd ict sector definition was originally approved in 1998. It was amended slightly in 2002 to reflect isic Rev. 3.1 changes to Wholesale." (La actual definición de la tic para la ocde se aprobó originalmente en 1998. Fue modificada levemente en 2002 para reflejar los cambios mayores en la isic Rev. 3.1 [traducción propia]). 
idea de que la tecnología se debería centrar en la recopilación y almacenamiento de datos más que en su aprovechamiento para el bienestar común. En contraste con este culto a la información, surge el término $\mathrm{TD}^{4}$ para poner de presente que el foco de las tecnologías radica en la relación entre estas y el modo en que las personas las usan, y no en su potencial "informativo" o "comunicativo".

En el presente artículo se sostiene que, por sí solas, las TD no posibilitan un cambio en la educación más allá de ciertos aspectos formales (la automatización de problemas y respuestas, el almacenaje y circulación de materiales). En cambio, son los actores quienes pueden transformar la educación, aprovechando sus propiedades innovadoras, siempre y cuando existan diseños (tecnológicos) adecuados y se resuelvan sus necesidades educativas.

Tal como ocurre con el término TD, la noción de acceso es determinante en el planteamiento de este trabajo ya que permite construir estrategias de acción matemática ante situaciones sociales usando la tecnología como medio que transforma comportamientos, los cuestiona, permite reflexionar en torno a ellos y, en última instancia, los modifica. Desde las teorías socioculturales y resignificando el término acceso para las prácticas de lengua escrita, Kalman (2004) propone que este "refiere a las oportunidades de participar en eventos de lengua escrita teniendo situaciones en las que el sujeto se posiciona frente a otros lectores y escritores" (2004, p. 26). Un uso ampliado de este término puede incluir las TD y, en consecuencia, visibilizar "las prácticas transformadoras con las cuales la gente hace significados identificables mediante el uso de tecnologías digitales" (Gillen y Barton, 2010, p. 9).

Una premisa para fomentar este acceso es tomar como punto de partida lo que los adultos saben, en lugar de lo que suponemos que ignoran, para posteriormente utilizar la tecnología como un puente hacia otras maneras de representar y acceder a ideas matemáticas poderosas y, posteriormente, a oportunidades de participar en eventos culturales. Esta visión de las TD como medio va en contra del determinismo -en donde se presupone que, por sí solas, estas herramientas impactan directamente en el desarrollo educativo de los sujetos- y vira la atención al estudio de la tecnología como herramienta que organiza el proceso de acceso a ideas matemáticas poderosas con el objetivo de resolver sus necesidades educativas.

Uno de los propósitos de la investigación referida era exponer la posibilidad de llevar a cabo una acción educadora matemática que incluyera el uso de tecnologías, que no estuviera tan disociada de las actividades matemáticas culturales, sino que, por el contrario, las retomara en la mayor

4 El término td se utiliza en la mayoría de las investigaciones en el campo de la matemática educativa. (Véanse por ejemplo las actas del xVı encuentro de la Comisión Internacional en Instrucción Matemática [ICMI] sobre Tecnología [Hoyles y Lagrange, 2010]).

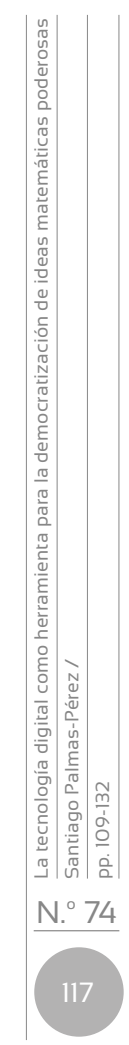


medida posible, utilizando el potencial dinámico de las TD. El interés se encuentra en el análisis del papel que pueden desempeñar las tecnologías en la EMDJA, retomando las concepciones matemáticas previas de los educandos, basándose en sus prácticas matemáticas sociales y vinculándolas con ideas matemáticas poderosas.

\section{Beneficios registrados del uso de las TD en la educación matemática}

Las maneras de expresar el conocimiento matemático varían dependiendo del medio de representación que se use. Por ejemplo, usar lápiz y papel permite ciertas acciones que las TD no y viceversa. Representar de distintas maneras los conocimientos matemáticos enfatiza y facilita el trabajo con diferentes secciones de los conceptos. Por ejemplo, en una hoja de cálculo, al notar los cambios dinámicos entre una tabla de datos y su representación gráfica podemos Ilamar la atención para que se note la pendiente de la gráfica y formular hipótesis acerca de cómo cambia la inclinación de la gráfica dependiendo del valor $m$ en $y=m x+b$. Otro ejemplo es la representación de la geometría que han suscitado los programas de geometría dinámica, que han cambiado la manera en que se interactúa con los contenidos.

Junto con una didáctica y un diseño de software adecuado, las TD podrían abrir la posibilidad de acceder a la matemática intrínseca en dichas representaciones y, en consecuencia, aprender interactuando con objetos matemáticos. En concreto, distintas formas de representar conocimientos matemáticos favorecen (o no) trabajar de cierta manera un concepto matemático; la representación y el modo de trabajar el concepto emergen simultáneamente.

Enfocándose en la educación matemática, la pluralidad de usos tecnológicos depende de los diseños y, más relevantemente, de las prácticas alrededor de estas tecnologías. Algunos usos de las TD constituyen nuevas formas de vincularse con ideas o conceptos, sobre todo aquellos en los que la actividad matemática cambia la manera en que tradicionalmente los educandos se relacionan con el conocimiento, lo cual genera nuevos vínculos entre la actividad humana y las representaciones modeladas por la tecnología. Las TD, sus entornos virtuales, usos, representaciones y herramientas pueden colaborar en la reflexión de ideas matemáticas poderosas tanto a nivel macro (institucional y social) como micro (didáctico y cognitivo).

A continuación, se mencionan algunas evidencias de los beneficios del uso de la TD en la educación matemática. Uno de ellos es recibir retroalimentación instantánea cuando se interactúa con objetos matemáticos, lo cual promueve que los educandos hagan conjeturas y sigan explorando (Clements, 2000), como cuando se ejecuta el programa Logo y se depura (Nastassi, Clements y Battista, 1990). Asimismo, se registra que al usar 
la tecnología para hacer dibujos geométricos se promueven procesos de experimentación y la formulación de conjeturas (Jarret, 1998; Ruthven y Hennessy, 2002); por ejemplo, al modificar los vértices de un triángulo en un programa de geometría dinámica y notar que la suma de los ángulos interiores es constante.

En América Latina, los estudios de Rojano (Butto y Rojano, 2004; 2010; Rojano, 2002, 2003), muestran cómo la introducción de la tecnología puede dar acceso a ideas y procesos de generalización matemática a temprana edad, de los 10 a los 11 años. A partir de estos estudios, se muestra la factibilidad de presentar a estudiantes de la escuela básica el pensamiento algebraico, aprovechando los tópicos de aritmética y las potencialidades del lenguaje de programación (Butto y Rojano, 2010, p. 83). Pensando específicamente en los aspectos sobre el numeralismo, el estudio de Geiger, Goos y Dole (2015) muestra cómo la TD, en el salón de clases, puede mejorar la forma en que se abordan elementos matemáticos, siempre y cuando se relacionen con contextos de la vida extraescolar.

Estos ejemplos de los beneficios del uso las TD corresponden, en su mayoría, a lo estudiado con la población de niños en edad escolar. Un buen reto $-\mathrm{y}$ parte de lo que interesa en el presente estudio- es el análisis de la posibilidad de que dichos beneficios puedan impactar a una población de jóvenes y adultos con baja escolaridad. Hay que señalar que los adultos poseen de antemano concepciones matemáticas que pueden ser potenciadas con el uso de las TD, además de que le otorgan un valor social al uso de dichas tecnologías.

\section{Desafíos del uso de las TD en la EMDJA}

La investigación sobre la incorporación de las TD en la EMDJA implica analizar algunos desafíos. Similar a lo que Rojano (2002) expone:

... también se puede decir que la incorporación de la tecnología de la información al salón de clases en donde se imparten matemáticas acarrea consigo la posibilidad de democratizar el conocimiento matemático [...], que previamente solo era accesible para una minoría de estudiantes que querían seguir una carrera universitaria científica. (Rojano, 2002, p. 158; traducción propia) $)^{5}$.

En mi posición, un reto de las TD en la EMDJA es justamente abrir la posibilidad de democratizar el conocimiento fuera del aula considerando procesos educativos que funjan como puentes entre lo que los adultos saben y lo que quieren conocer.

5 "[...] it also can be said that the introduction of information technology to the mathematics classroom has brought with it the possibility of democratizing mathematical knowledge [...], which previously was only accessible to a minority of students wanting to follow a scientific university degree." (Rojano, 2002, p. 158). 
Si los diseños tecnológicos pueden lograr que en las TD se plasmen desafíos cognitivos adecuados para adultos usando el potencial de la tecnología, es posible pensar en eventos educativos para la EMDJA que den lugar a procesos de democratización de ideas matemáticas que no infantilicen cognitivamente las actividades. Dichos retos se pueden diseñar por medio de la manipulación directa de objetos ejecutables dentro de las TD, manteniendo propiedades matemáticas y fomentando su uso desde una situación didáctica.

Desde la mirada del numeralismo, en la que se visibiliza un proceso mediado por la sociedad en el que se construye una cultura matemática con base en la articulación entre acciones culturales y las matemáticas se abre la posibilidad de distinguir estas últimas como prácticas sociales y, sobre todo, como una acción cultural. Esta visión coincide con la de Hoyles y Noss $(2003,1996)$ con respecto a distinguir las matemáticas como actividad. Metodológicamente, "[...] podemos introducir nuevas nociones y tratar de entender cómo una persona pensante las relaciona con lo que ya conoce ${ }^{\prime \prime}$ (Noss y Hoyles, 1996, p. 9; traducción propia). Pretender que los adultos con baja escolaridad aprendan las matemáticas -formales, institucionales y determinadas por ciertas reglas algebraicas- se puede sustituir por la intención de que desarrollen y pongan en práctica actitudes matemáticas como la creación de hipótesis, la experimentación, la corroboración y la comparación entre ideas; todo esto con base en el análisis de las necesidades académicas que los educandos identifiquen.

Al concebir las matemáticas como actividad, se crea un campo abierto para la enseñanza de procesos de modelización (y generalización), sin tener que elaborar constructos abstractos o un lenguaje formal algebraico "desde cero". En vez de ello, las interacciones entre lo concreto y abstracto se toman como eje de la idea matemática de modelización. Como se verá, en el diseño de las aplicaciones tecnológicas y el diseño didáctico correspondientes se da preferencia al incentivo de actitudes matemáticas, y con ello a la interacción entre lo concreto y lo abstracto mediado por la tecnología. Esta interacción constituye, en sí, la manera en que se concibe el aprendizaje ${ }^{7}$ en el presente trabajo: un aprendizaje definido por la modificación de conocimientos y saberes, posterior a la puesta en práctica de conocimientos. En el momento de poner en práctica las concepciones previas dentro de una situación de conflicto cognitivo se aprende y, posteriormente, a partir de las experiencias de aprendizaje se construyen ideas matemáticas que pueden o no ser significativas (dependiendo de las dificultades experimentales y de las características del educando).

6 " $[\ldots]$ we can introduce new notions and try to understand how the thinker connects these with what he or she already knows".

7 Basado en el constructivismo, específicamente en la definición de Brousseau, según la cual "el sujeto aprende corrigiendo sus acciones y anticipando sus efectos" (Brousseau, 2007, p. 54). 
Es una situación de conflicto inspirada en el conflicto cognitivo de Piaget en donde el educando se replantea sus esquemas y transita hacia otra estructura cuestionando la anterior.

El reto de crear un material usando las TD para la EMDJA implica reflexionar en torno a las características de un software matemático. Al respecto, Noss y Hoyles (1996) señalan la necesidad de que este se convierta en una ventana de representaciones y procesos matemáticos. En teoría, este tiene que poner una arena en la que se pongan en juego las representaciones de las concepciones matemáticas del educando -en este caso un adulto con baja escolaridad-, se creen conflictos, se retroalimente adecuadamente y se aprenda: "Un Software que no proporcione al alumno medios de expresión de ideas matemáticas no abre ninguna ventana a los procesos de aprendizaje de las matemáticas" (Noss y Hoyles, 1996, p. 54; traducción propia).

Idealmente, el software matemático cambia las representaciones comunes del conocimiento y las presenta, manipula y desarrolla de otra manera (DiSessa, 1988, p. 3). Esta diferencia consiste en cambiar la manera en que, semióticamente, se representa y se interactúa con el conocimiento matemático. El diseñador del software puede deliberadamente presentar un conocimiento matemático de tal manera que el educando construya la noción actuando sobre ella, tal como lo señalaban Noss y Hoyles (1996). En concreto, lo que deja esta teoría de ventanas a conocimientos matemáticos (Noss y Hoyles, 1996), es que el desarrollo del software y las situaciones didácticas idealmente confrontan las representaciones y organizan el tránsito entre concepciones previas al momento educativo e ideas matemáticas poderosas.

Al usar la computadora, los adultos pueden contrastar las representaciones matemáticas que han usado hasta el momento con las que se muestran en ella. Entonces la tecnología entra como el medio (al estilo Brousseau) que crea las posibilidades para que el educando confronte sus nociones previas y por lo tanto modifique los conocimientos y aprenda. Así, se concibe la tecnología como un puente hacia ideas matemáticas poderosas.

En concordancia con la posición de que por sí solas las TD no generan un cambio, es importante que el desarrollo tecnológico se acompañe de una situación didáctica que la organice. Las secuencias didácticas tratan de fomentar que los educandos creen "anticipaciones" acerca de ciertos problemas. Estas anticipaciones son diferentes formas de representar y tratar el conocimiento y, por lo tanto, señales de aprendizaje. Por ejemplo, si el adulto tiende a pensar que el área de cualquier superficie se puede calcular siempre como "base por altura", la tecnología y la situación

8 Sin excluir el papel del educador. 
didáctica mostrarán que hay figuras en las que dicha fórmula es menos precisa y, por lo tanto, tendrá que modificar su pensamiento y crear una estructura de pensamiento relativa a la anticipación.

Uno de los retos para la EMDJA es la redefinición de las prácticas educativas de adultos con relación al acceso a las TD. Esto abre la posibilidad de construir una nueva forma de entender los procesos educativos de esta población en cuanto a aspectos sociales, económicos y culturales. Para los educadores e investigadores dedicados a las matemáticas para esta población, el reto es concebir la tecnología como una herramienta útil en el espacio educativo para los procesos de enseñanza y aprendizaje de las matemáticas de jóvenes y adultos.

\section{Posibilidades del uso de las TD en la EMDJA}

La falta de estudios sobre el uso de las TD y la EMDJA deja entrever un campo fértil de investigación en varias líneas. El primero es que, al no estar plenamente identificado el campo educativo de la EMDJA, los usos de las TD pueden ser reportados dentro de otros campos (por ejemplo: educación rural, educación para el trabajo, migración) y no es posible identificarlos como parte del campo educación de adultos y analizarlos en conjunto. Por otro lado, el puente que pueden tender las TD como vías de acceso a ideas matemáticas parte de la democratización del conocimiento.

A partir de lo anterior, se plantean tres argumentos de lo que las TD aportan a la EMDJA:

1. Las TD generan nuevas formas de expresar conocimiento matemático y por lo tanto nuevas formas de actuar sobre este, por lo que abren la posibilidad de tender un puente entre acciones matemáticas culturales e ideas matemáticas poderosas, haciendo uso de la mediación, en una doble tarea de representación, tanto de las acciones culturales como de los modelos matemáticos. Esta concepción es la de la TD como una herramienta de vínculo entre lo concreto y lo abstracto.

2. Cuando la tecnología se concibe como un mediador cultural entre el sujeto y el conocimiento, esta modifica la relación que tienen los educandos con conceptos e ideas matemáticas, y por lo tanto la tarea didáctica y la acción cognitiva. En consecuencia, es posible crear un diseño didáctico y tecnológico que posicione al educando como un agente ante una cultura matemática, y le permita experimentar de primera mano ideas matemáticas que, sin la tecnología, existirían solo en una dimensión teórica.

3. El potencial que tiene la tecnología de abrir canales de comunicación con conceptos e ideas matemáticas poderosas. Es decir, con 
la mediación de las TD es posible discutir y actuar sobre ideas matemáticas poderosas y, por lo tanto, democratizar el conocimiento.

A continuación se muestra un ejemplo de cómo la tecnología puede desempeñar un papel como puente, como herramienta que amplía la manera en que se representa e interactúa con el conocimiento matemático y, sobre todo, como herramienta que posibilita la democratización de ideas matemáticas poderosas ante necesidades educativas específicas.

\section{Ejemplo de herramienta de acceso a ideas matemáticas poderosas}

El presente ejemplo surge de una investigación en la que se analiza el papel de las TD en el campo de la EMDJA de baja escolaridad. La manera en que se abordó la cuestión fue desarrollando un diseño didáctico basado en la teoría de situaciones didácticas (Brousseau, 1997), así como una herramienta tecnológica ad hoc que funcionara como puente hacia ideas matemáticas poderosas.

\section{Análisis preliminares}

Para comenzar, se hicieron una serie de entrevistas con cinco adultos que no han completado su educación básica, registrando sus necesidades educativas ${ }^{9}$.

\section{Necesidades educativas geométricas de adultos}

El tema de cálculo de superficies surgió a partir de la petición de varios adultos que tenían dificultades para calcular superficies que no fueran rectángulos. Por ejemplo:

Entrevistador: [...] Si necesitas por ejemplo medir, eh, decías que aplanar, calculaban los metros cuadrados, ¿no? Es diferente calcular los metros cuadrados en una pared, o en un techo o abajo, ¿o es lo mismo siempre?

Claudia: Pues es lo mismo, o sea medir el techo o la pared, bueno yo lo mido, ahora sí que lo largo por lo ancho.

Así como Claudia, todos los demás participantes calculaban el área de cualquier superficie multiplicando el ancho por lo largo, fuese un triángulo, círculo o lo que fuese. El sobrante era parte "natural" del cálculo y se contemplaba con pérdidas o excedentes de material.

9 Aunque existieron otro tipo de necesidades educativas, en este trabajo solo expondré las geométricas. 
En el siguiente extracto se puede ver cómo una de las mujeres entrevistadas tiene una necesidad de entender cómo se calcula el dinero que se cobra por aplanar un piso:

Yeni: Bueno, mi marido es herrero y él, nomás viendo, lo sacó. Y yo me quedo: ¿de dónde lo viste o cómo lo viste? Explícame por qué. Igual los centímetros cúbicos de las casas. Si he visto con uno de mis tíos que es albañil y digo ¿cómo le sacan eso?, ¿no?

Entrevistador: Eso de cubicar ¿no?

Y: Ajá. Que le cobro por metros aplanados y, por tanto. O por la loseta, tanto cobro, 4 por 4 . Ah ok, sólo ellos entienden de qué hablan. No, la verdad, ni idea tengo.

Lo que nos cuenta Yeni refleja su interés por conocer ciertas nociones matemáticas para poder participar de ellas. El aprender cómo se miden las superficies de "las casas" y por lo tanto cuánto se cobra por un trabajo podría promover su participación en ciertas actividades laborales de las que hasta el momento se encontraba al margen.

El cálculo de superficies apareció también en la petición de Felipe:

Entrevistador: Pero, por ejemplo, en su vida cotidiana, en su trabajo, ¿ha requerido de algunas matemáticas que no haya podido hacer?

Felipe: Sí [...] Bueno, aquí en mi trabajo una vez necesité saber el volumen de agua que teníamos almacenado en la cisterna. Entonces yo no supe cómo cubicar y sí me quedé un poquito frustrado y tuve que preguntarle a un maestro de matemáticas y circuitos, fíjate. No sé cuánta cantidad de agua le entra a una cisterna. Entonces ya más o menos me dijo: multiplica tanto por tanto y luego da, para que salgan los metros cúbicos. Y solo así se arregló el problema. Obvio, yo a mi jefe directo no le dije: no sé. Sino que me dio pena, me sentí frustrado y yo le dije: no sí, yo le digo al rato. Entonces recurrí a un maestro y ya cuando... ah pues fueron 80 metros, ah que bueno me da gusto que sepa hacerlo, pero no, él no sabía que había ido a preguntar.

La necesidad educativa se registra en el momento de la entrevista cuando Felipe nos muestra que, al no poder calcular el volumen de agua necesaria para Ilenar una cisterna, se siente frustrado. De nuevo aparece la necesidad de manejar el cálculo de superficies y volúmenes para poder participar en una actividad laboral. Otras necesidades educativas encontradas en las entrevistas fueron: 1) calcular el área de alguna pared para cobrar por metro cuadrado de aplanado y 2) para calcular el área de cierto terreno y pedir financiamiento (fertilizante o cemento). 
Ante esta situación, se planteó una alternativa a la educación tradicional donde se enseña una fórmula para cada figura geométrica (una fórmula para el cuadrado, otra para el triángulo, etcétera). En contraste, en el presente trabajo se eligió explorar el uso del teorema de Pick como fórmula única con la que se calcula el área de polígonos con vértices enteros.

El teorema de Pick dice:

Sea un polígono simple cuyos vértices tienen coordenadas enteras. Si $B$ es el número de puntos enteros en el borde, I el número de puntos enteros en el interior del polígono, entonces el área A del polígono se puede calcular con la fórmula:

$$
\mathrm{A}=I+\frac{\mathrm{B}}{2}-1
$$

Tradicionalmente, el currículo escolar (por ejemplo, en México) propone, para el cálculo del área de figuras irregulares, el método de triangulación. El problema es que en muchas ocasiones se necesita el teorema de Pitágoras o trigonometría para calcular el área de los triángulos y esto solo se enseña a partir de tercero de secundaria; es decir, las personas sin educación básica no lograrían construir este conocimiento. En contraste, el teorema de Pick cambia la aproximación cognitiva al cálculo del área usando aritmética básica y el conteo como base para el cálculo del área, acercando la posibilidad de calcular figuras irregulares. Se pensó en este teorema por los siguientes beneficios para la población específica con la que se trabaja:

» Uso y sistematización de concepciones matemáticas previas de los adultos.

» Acceso al cálculo del área de un gran repertorio de figuras irregulares.

» Poca memorización de fórmulas.

Así, el teorema surge como una opción que proviene del mundo matemático institucional; sin embargo, conscientes del desafío que implica usar el teorema de manera aislada, se creó un diseño tecnológico ad hoc y una secuencia didáctica para organizar los momentos educativos con los educandos. 


\section{Diseño tecnológico}

La "calculadora de superficies" consiste en una cuadrícula en la que se pueden colocar vértices hasta cerrar una figura. Al hacerlo, aparecen los puntos interiores y puntos en el perímetro con coordenadas enteras (véase la figura 1).

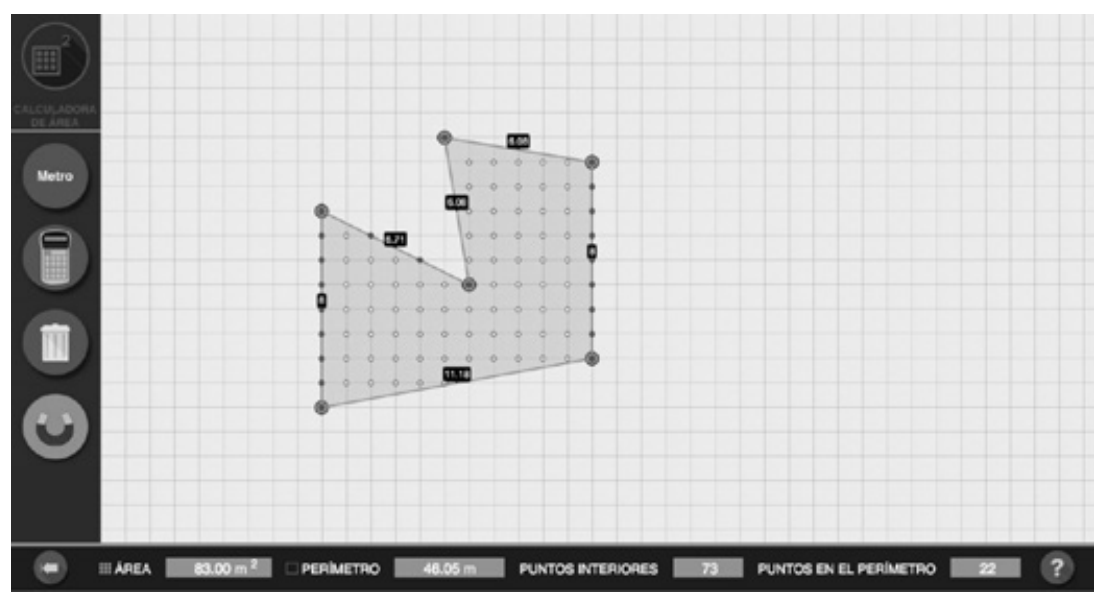

Figura 1. Calculadora de superficies

Didácticamente, la necesidad de crear un ambiente digital emerge de la representación gráfica que el teorema fomenta. Por ejemplo, observar en tiempo real dónde y cómo se colocan los puntos interiores y en el borde al cerrar la figura. Con esta herramienta ad hoc es posible editar la posición de los vértices, y así reconocer cómo, a partir de estos cambios, el área o el perímetro cambian dinámicamente.

\section{Diseño didáctico}

La secuencia didáctica comienza con un "círculo de cultura" (Freire, 1970) en el que se promueve la reflexión sobre diferentes temas de aspecto social que problematizan la puesta en práctica de la tecnología en la educación. Estos temas son extremadamente sensibles para los adultos con los que se trabaja, pero tres ejemplos pueden ser: 1) la problematización sobre la diferencia de la educación para niños y la educación de adultos, 2) la tecnología como creación del ser humano para el ser humano, o 3) la reflexión sobre el aprendizaje significativo en comparación con el aprendizaje por conceptos aislados. Estos temas engloban toda la secuencia didáctica y le dan el sentido social que merece la EMDJA.

Posterior al diálogo, se comienza con un problema generador en el cual se pide calcular el área de la siguiente figura de cualquier manera posible (Veáse la figura 2). 


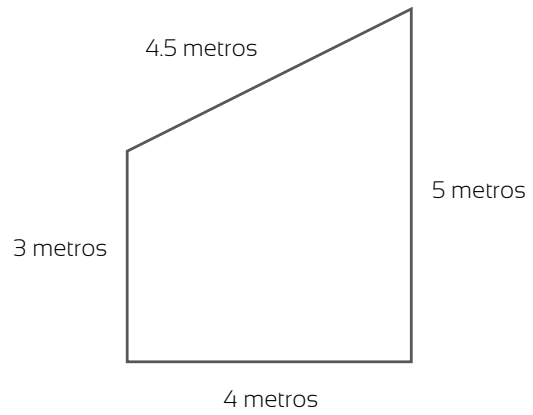

Figura 2. Problema generador

Este problema está basado en dos antecedentes académicos: Agüero (2006) y Estrada y Ávila (2009), que lo propusieron como un problema de exploración del cálculo de área con adultos de baja escolaridad. Los siguientes pasos didácticos son:

» Presentación escrita y oral del teorema de Pick.

» Uso del teorema y de la aplicación tecnológica ad hoc con rectángulos que sirven para constatar la concepción previa de $b^{\prime} h$.

» Determinar la fórmula del triángulo experimentando en la aplicación tecnológica.

» Cálculo de áreas de figuras regulares.

» Cálculo de áreas de figuras irregulares.

»Estimación como proceso de cálculo de problemas en la vida cotidiana.

» Problemas contextualizados.

» Regreso al problema generador.

Cada uno de estos pasos alude a elementos teóricos de la teoría de situaciones didácticas (Brousseau, 1997), como la institucionalización, la validación, la devolución y la formulación dialéctica. Esta secuencia didáctica abarca, aproximadamente, tres sesiones de dos horas cada una.

\section{Tecnología como puente hacia ideas matemáticas poderosas}

Al utilizar la herramienta tecnológica junto con la secuencia didáctica se registraron los siguientes resultados con respecto a la tecnología vista como puente hacia ideas matemáticas poderosas: 


\section{Del dibujo a la figura geométrica}

Se registró que el estudio de nociones como área y perímetro requiere construir un puente entre el dibujo y la figura geométrica. Esto ocurrió durante la secuencia didáctica en el momento que se comenzaba a analizar paredes o terrenos para posteriormente esquematizarlos en una figura geométrica. Algo interesante es que los propios educandos reconocían que al modelar los terrenos no se podía obtener un cálculo exacto por pequeños errores intrínsecos a la medición. Asimismo, algo digno de resaltar es que, al analizar una figura geométrica que se pueda inscribir en una rejilla, surge la importancia de analizar cómo distinguir si un punto es latiz o no, es decir, si tiene coordenadas enteras. Este hecho hace pensar que, en una evolución de la secuencia didáctica, el siguiente paso tendría que ser un análisis sobre cómo decidir si un punto sobre una línea tiene coordenadas enteras dado un segmento con vértices enteros.

\section{Del método de cálculo específico a métodos generales}

Al comenzar con los estudios preliminares, se registró que los adultos construían maneras de calcular el área de figuras irregulares usando el conocimiento de $b^{\prime} h$. Aunque con este conocimiento el repertorio de figuras es amplio, el error es grande para aquellas que no se asemejen a rectángulos. Aceptar la idea de que se puede calcular el área de cualquier polígono cuyos vértices se puedan colocar una retícula mediante el teorema de Pick refina el cálculo haciendo del conocimiento $b^{\prime} h$ un método más por elegir dentro de un acervo de concepciones matemáticas previas de los adultos. El acceso a este conocimiento se logró a través de un desarrollo virtual de aprendizaje conformado por una situación didáctica y un desarrollo ad hoc. La elección del teorema de Pick funcionó como un puente entre métodos de cálculo específicos y métodos generales, en gran parte por el análisis de las concepciones previas (sobre todo los métodos de cálculo previos que se encontraron en la exploración de concepciones matemáticas previas); y, en particular, la aritmética básica que utiliza el teorema, la sencilla expresión en una ecuación y su expresión gráfica en el desarrollo ad hoc.

En general, los retos cognitivos que tuvieron los educandos con la herramienta y la secuencia didáctica se encaminan hacia un pensamiento más abstracto en el que es posible calcular el área de un mayor número de figuras, abstrayendo la idea de que la figura existe dentro de una retícula y se pueden contar sus puntos interiores y puntos en el borde dependiendo de las medidas de los lados. Además de esto, se registró una construcción del significado de términos como área y perímetro. 


\section{Conclusiones}

La secuencia didáctica, junto con la herramienta tecnológica, lograron que los educandos no solo construyeran y utilizaran nuevos conceptos, sino ideas matemáticas poderosas. Dicha construcción de conocimiento surgió de no infantilizar los retos cognitivos que se planteaban en la secuencia, por ejemplo, el usar una fórmula algebraica para presentar un teorema a adultos con baja escolaridad (varios de ellos sin educación secundaria). Los adultos tienen la suficiente capacidad cognitiva y el interés como para lidiar con conceptos y representaciones trabajados durante la secuencia didáctica. La posición teórica tomada en esta investigación plantea el punto de partida de un proceso educativo con adultos de baja escolaridad desde lo que sí saben y no desde lo que suponemos que ignoran.

Ante la invisibilización de los procesos educativos incorporando la tecnología para la EMDJA, el estudio que se presenta en este artículo se posiciona como una oportunidad de analizar la potencialidad de las TD creando un diseño didáctico y tecnológico ad hoc específico para esta población como herramienta de democratización del conocimiento. Distinguir la tecnología como herramienta ayuda a ver dos aspectos importantes: la parte social de introducción de una herramienta ajena a lo cotidiano y el uso expedito que se les da a dichos instrumentos. Concebir la tecnología como medio hará que se caractericen sus posibilidades didácticas, por ejemplo, planeando cómo el educando confrontará sus saberes matemáticos previos y analizando cómo podría funcionar la acción de retroalimentación del medio hacia el educando.

En este artículo se mostró la posibilidad de que la tecnología ofrezca un acceso democrático a ideas que habitualmente estaban limitadas a personas en procesos escolares. En este caso, los adultos tienen acceso a ciertas ideas matemáticas poderosas por medio del desarrollo de un programa ad hoc y de la secuencia didáctica construidas a partir de sus necesidades educativas. Que la tecnología funja como herramienta democratizadora se favorece tanto por el diseño ad hoc, es decir, la manera en que se permite manipular objetos matemáticos, como por el diseño didáctico que organiza la situación, pero, sobre todo, por la manera en que los adultos se relacionan con dicha tecnología, que responde a sus necesidades educativas. 


\section{Referencias}

Agüero, M. (2006). El pensamiento práctico de una cuadrilla de pintores. Estrategias para la solución de problemas en situaciones matematizables de la vida cotidiana. Pátzcuaro, Michoacán, México: crefal-Universidad Iberoamericana.

Brousseau, G. (1997). Theory of didactical situations in mathematics. (M. C. Nicolas Balacheff, ed.) Dordrecht, Netherlands: Kluwer Academic Publishers.

Brousseau, G. (2007). Iniciación al estudio de la teoría de las situaciones didácticas. (D. Fregona, trad.). Buenos Aires, Argentina: Libros del Zorzal.

Butto, C. y Rojano, T. (2004). Introducción temprana al pensamiento algebraico: abordaje basado en la geometría. Educación Matemática, 16(1), 113-148.

Butto, C. y Rojano, T. (2010). Pensamiento algebraico temprano: El papel del entorno Logo. Educación Matemática, 22(3), 55-86.

Camargo, L., y Sandoval, I. (2017). Acceso equitativo al razonamiento científico mediante la tecnología. Revista Colombiana de Educación, (73), 179-211.

Castells, M. (1996). La era de la información. Economía, sociedad y cultura. Ciudad de México, México: Siglo xxi.

Castells, M. (2000). The rise of the network society (2d ed.). Malden, Mass.: Blackwell.

Clements, D. (2000). From excercises and tasks to problems and projectUnique contributions of computers to innovative mathematics education. The Journal of Mathematical Behavior, 19(1), 9-47.

D’Ambrosio, U. (1996). Educacão matemática: Da teoria à pràtica. Campinas, Brasil: Papirus.

DiSessa, A. (1988). Social niches for future software. En A. diSessa, M. Gardener, J. Greeno, F. Reif, A. Schoenfeld \& E. Stage. Towards a Scientific Practice of Science Education. New Jersey, NY: Lawrence Erlbaum.

Estrada, J. L. y Ávila, A. (2009). Los usuarios de la educación básica para jóvenes y adultos y la solución de un problema de área. Educación Matemática, 21(3), 33-66.

Flecha, R. (1999). New educational inequalities. En M. Castells, Critical Education in the New Information Age (pp. 65-82). Cumnor Hill, Oxford, England: Rowman \& Littlefield.

Freire, P. (1970). Pedagogía del oprimido (30. a ed.). México: Siglo Veintiuno Editores. 
Geiger, V; Goos, M. y Dole, S. (2015). The role of digitial technologies in numeracy teaching and learning. (N. S. Council, ed.) International Journal of Science and Mathematics Education, 1115-1137.

Gillen, J. y Barton, D. (2010). Digital Literacies. A Research Brifing by the Technology Enhanced Learning phase of the Teaching and Learning Research Programme. Londres: London Knowledge Lab, University of London.

Hoyles, C. y Lagrange, J.-B. (2010). Mathematics education and technologyrethinking the terrain. Nueva York, Dordrecht, Heidelberg, Londres: Springer.

Hoyles, C. y Noss, R. (2003). What can digital technologies take from and bring to research in mathematics education? Second international handbook of mathematics education (pp. 323-349). Dordrecht: Springer.

Jarret, D. (1998). Integrating technology into middle school mathematics. It's just good teaching. Northwest Regional Educational Laboratory.

Kaput, J. J. (1994). Democratizing access to calculus: New routes using old roots. (A. Schoenfeld, ed.). Mathematical Thinking and Problem Solving (pp. 77-155). Hillsdale, NJ, USA: Lawrence Erlbaum.

Laursen, K. (2004). New and old economy: The role of ict in structural change and economic dynamics. Structural Change and Economic Dynamics Special Issues, 241-243. Dorchester, Reino Unido: The Dorset Press.

Nastassi, B. K.; Clements, D. H. y Battista, M. T. (1990). Social cognitive interactions, motivation, and cognitive growth in Logo programming and cai problem-solving environments. Journal of Educational Psychology, 82, 150-158.

Noss, R. y Hoyles, C. (1996). Windows on mathematical meanings: Learning cultures and computers. Netherlands: Mathematical Education Library. Kluwe Academic Publishers.

Rojano, T. (2002). Mathematics Learning in the junior secondary school: Students' Access to significant mathematical ideas. (L. D. English, ed.). Handbook of international research in mathematics education (pp. 143-164). New York: Routledge.

Rojano, T. (2003). Incorporación de entornos tecnológicos de aprendizaje a la cultura escolar: proyectos de innovación educativa en matemáticas y ciencias en escuelas secundarias públicas en México. Revista Iberoamericana de Educación OEl, España 33, 135-169.

Ruthven, K. y Hennessy, S. (2002). A practitioner model of the use of computer-based tools and resources to support mathematics teaching and learning. Educational Studies in Mathematics, 49(1), 47-88. 
Schmelkes, S. y Kalman, J. (1996). La educación de adultos: estado del arte. Hacia una estrategia alfabetizadora para México. (inea, ed.) df, México: Instituto Nacional para la Educación de los Adultos.

Simon, H. (1977). What computers mean for man and society. Science, 195, 1186-1991.

Skovsmose, O. (1999). Hacia una filosofía de la educación matemática crítica. (P. Valero, trad.) Bogotá: Una empresa docente-Universidad de los Andes.

Skovsmose, O. y Valero, P. (2009). Democratic access to powerful mathematical ideas. En L. D. English, Handbook of International Research in Mathematics Education. (pp. 383-408). Nueva York: Routledge.

Street, B.; Baker, D. y Tomlin, A. (2008). Navigating numeracies: Home/ school numeracy practices. London: Springer-Science.

Valero, P.; Andrade-Molina, M. y Montecino, A. (2015). Lo político en la educación matemática: de la educación matemática crítica a la política cultural de la educación matemática. Revista Latinoamericana de Investigación en Matemática Educativa. 18(3), 287-300. Ciudad de México: Colegio Mexicano de Matemática Educativa A. C.

Warschauer, M. (2003). Technology and social inclusion: Rethinking the digital divide. Massachusetts: MIT. 